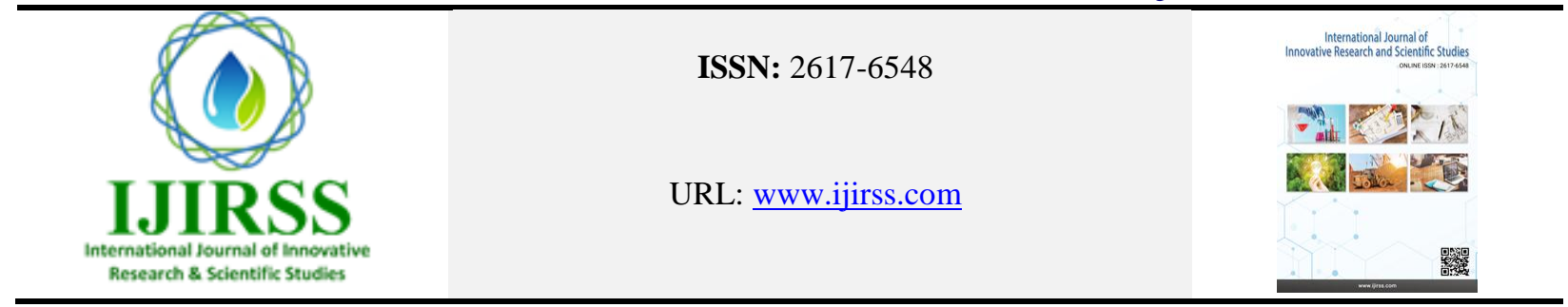

\title{
Barriers Role of Zero-Divisor Graph in Power Set Ring
}

\author{
Abdul Jamil Nazary \\ Department of Mathematics, Faculty of Education, Takhar University, Takhar, Afghanistan \\ (Email: jamil.nazary0030@gmail.com)
}

\begin{abstract}
This review presents the high performance of failure-resistant structural device system for the sustainable and flexible buildings. Firstly, the motivation and basic principles as well as methodology of the developing device system are explicitly illustrated. Then, the structural detail and seismic response of base isolation systems, namely, lead Rubber Bearing (LRB), HDLRB isolators, viscous damper base isolator (BIPD) and sliding bearing isolator (BISS) are summarized. The theoretical and experimental study results was shown that all four types of isolator system can be able to minimize damage after seismic an earthquake to the structural system. The viscous damper devices and energy dissipate as well as viscoelastic and fluid viscous dampening can be able to enhance the energy dissipation capacity of structural system under an earthquake loading. A placement of $\mathrm{L}$ shape, shear walls at the structural configuration plan is given more efficient behavior under seismic load than all other placements of shear walls at the building's configuration. Many numerical specimens of tunnel form buildings were constructed and modelled to analyze and interpreted the dynamic and static cyclic response of structures against seismic force. The deformation of the dynamic response of tunnel form building was smaller by using Carbon Fiber Reinforced Polymer (CFRP) repairing and retrofitting method. Contradictory, the usage of base isolations, energy dissipation devices, shear walls and tunnel form buildings can enhance the efficiency of structures under seismic force by reducing the economic cost saving in their construction.
\end{abstract}

Keywords: Base Isolator, Shear Walls, Tunnel Form Buildings, Passive Energy Dissipates.

DOI: $10.53894 /$ ijirss.v4i3.75

Funding: This study received no specific financial support.

History: Received: 24 March 2020/Revised: 2 June 2021/Accepted: 17 June 2021/Published: 30 June 2021

Licensed: This work is licensed under a Creative Commons Attribution 4.0 License $(\mathrm{ccc}) \mathrm{EY}$

Competing Interests: The author declares that there are no conflicts of interests regarding the publication of this paper.

Transparency: The author confirms that the manuscript is an honest, accurate, and transparent account of the study was reported;

that no vital features of the study have been omitted; and that any discrepancies from the study as planned have been explained.

Ethical: This study follows all ethical practices during writing. 
مقاله تحقيقى

\title{
نقش گَراف قاسمهاى صفر در رينگ ست طاقت
}

\author{
عبدالجميل نظرى \\ دييارتمنت رياضس، دانشكده تعليم وتربيه، د/نشكاه تخار، تخار، افغانستان \\ خلاصه
}

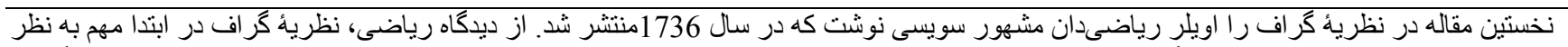

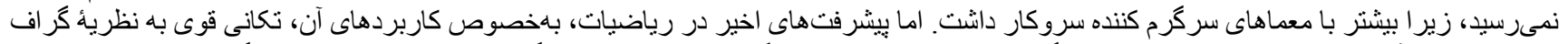

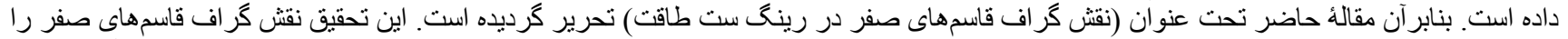

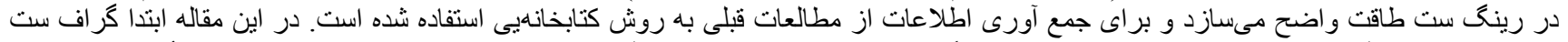

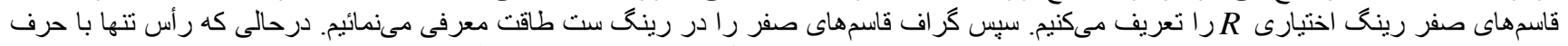

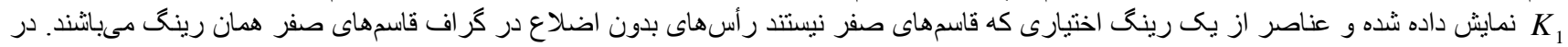

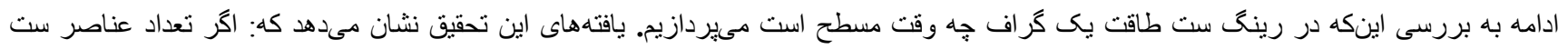
X

$$
\text { رينگ } P(X) \text { مسطح نمىباشد. }
$$

كلمات كليبى : ست، كر اف، قاسمهاى صفر، كر اف قاسمهاى صفر

1

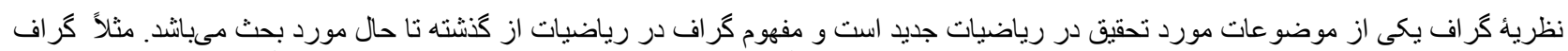

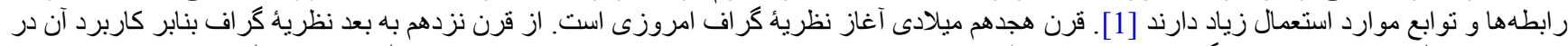

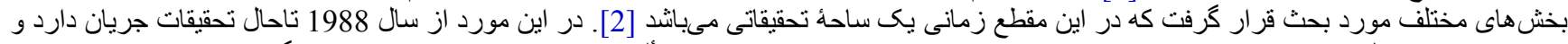

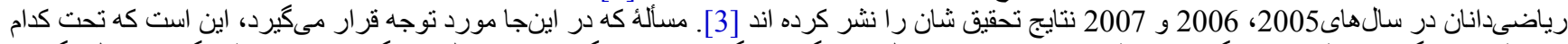

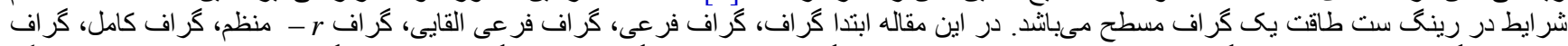

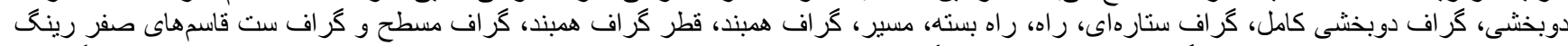

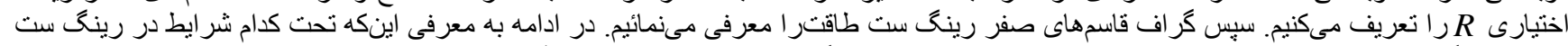

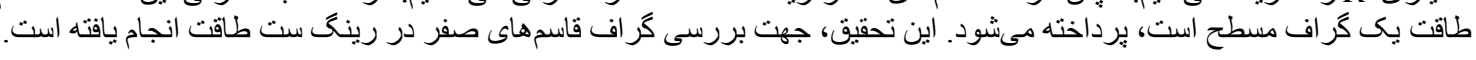

2.

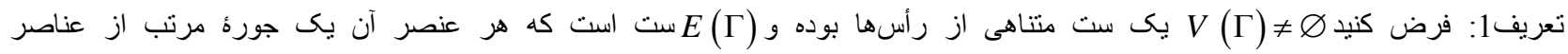

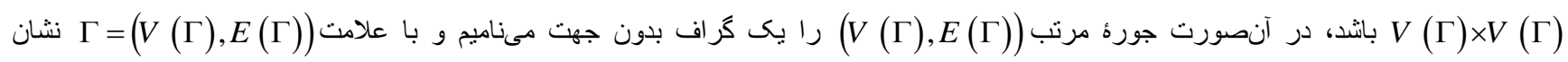

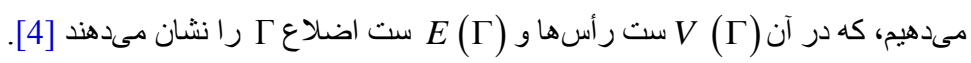

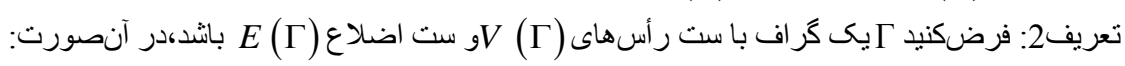

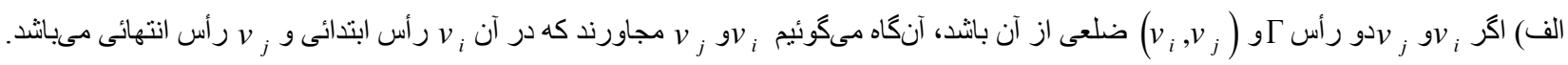

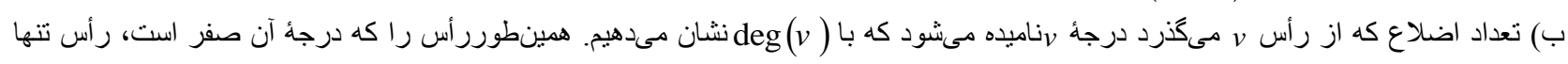

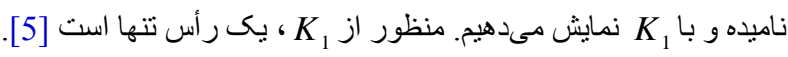

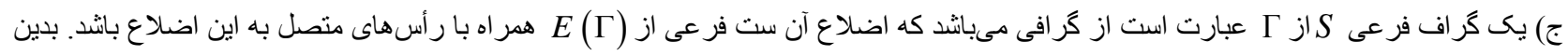

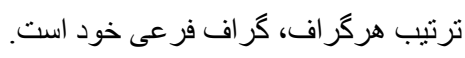

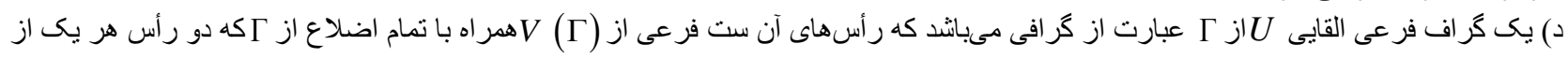

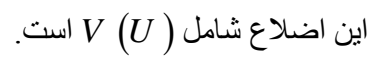

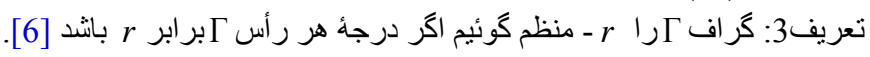

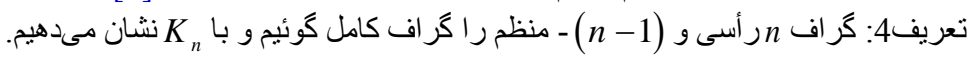
تعريف5: كراف $\Gamma$ را دو بخثى كوئيم هركاه

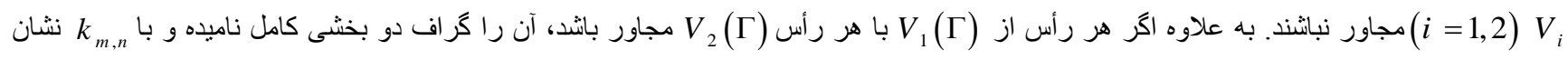
مىدهيم.كه در آن m

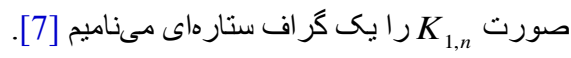




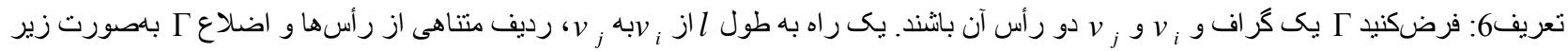

$$
\begin{aligned}
& v_{i}=u_{0}, e_{1}, u_{1}, e_{2}, u_{2}, e_{3}, \ldots, e_{l}, u_{l}=v_{j} \\
& v_{i}=u_{0}, e_{1}, u_{1}, e_{2}, u_{2}, e_{3}, \ldots, e_{l}, u_{l}=v_{j}
\end{aligned}
$$

به قسمىكه , بر

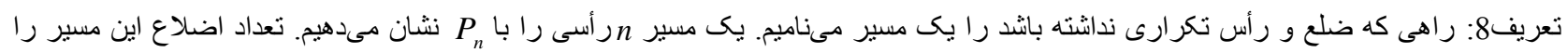

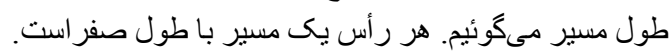

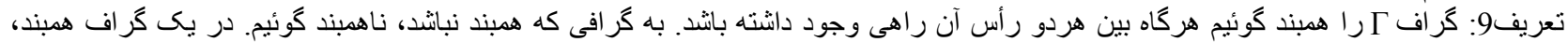

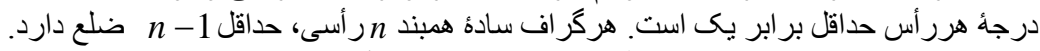

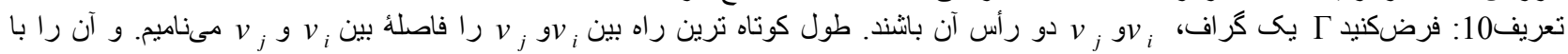

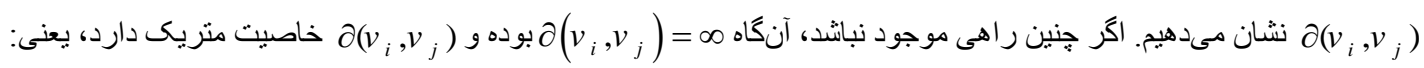

$$
\begin{aligned}
& \text { الف) همواره، } \\
& \text { ، } \partial\left(v_{i}, v_{j}\right)=\partial\left(v_{j}, v_{i}\right)( \\
& . \partial\left(v_{i}, u_{l}\right) \leq \partial\left(v_{i}, v_{j}\right)+\partial\left(v_{j}, u_{l}\right)(ج
\end{aligned}
$$

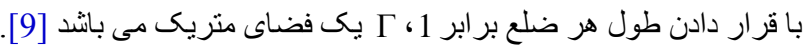

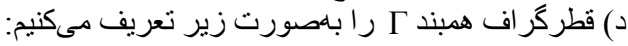
$\operatorname{diam}(\Gamma)=\max \{\partial(x, y) \mid x, y \in V(\Gamma)\}$

[10] diam

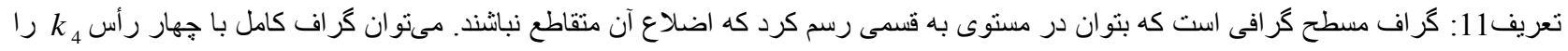

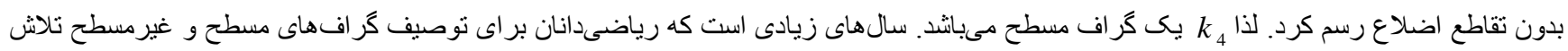

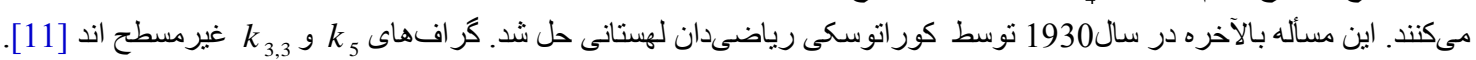

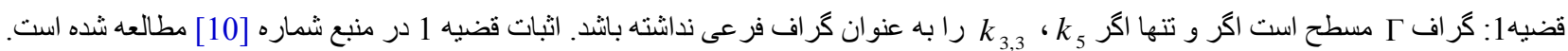

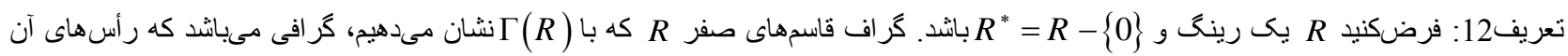

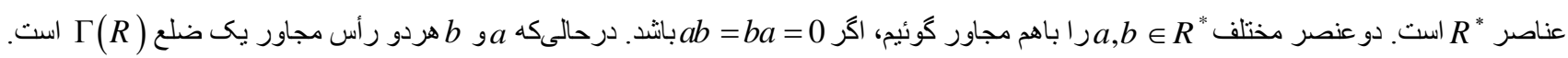

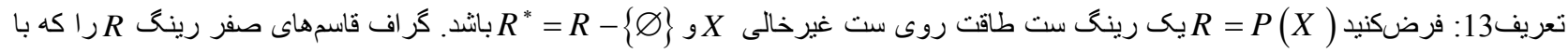

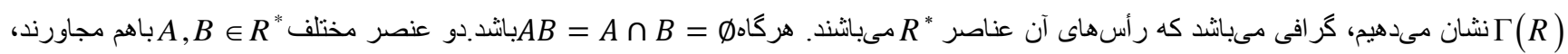

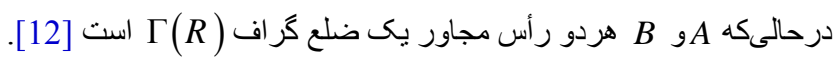

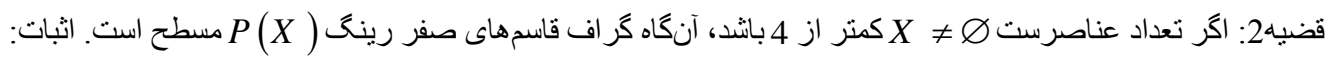
الف) اكر $X=\{a\}$

$$
\begin{aligned}
& R=P(X)=\{X, \varnothing\} \Rightarrow R^{*}=R-\{\varnothing\}=\{X\} \\
& \Rightarrow \Gamma(R)=K_{1}
\end{aligned}
$$

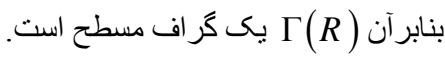

$$
\begin{aligned}
& \text { ب) اكر } \\
& R=P(X)=\{\{a\},\{b\}, X, \varnothing\} \\
& \Rightarrow R^{*}=R-\{\varnothing\}=\{\{a\},\{b\}, X\} \Rightarrow \Gamma(R)=P_{2} \cup K_{1} \\
& \text { بنابر آن } \\
& \text { ج) اكر }
\end{aligned}
$$

$R=P(X)=\{\{a\},\{b\},\{c\},\{a, b\},\{a, c\},\{b, c\}, X, \varnothing\}$

$$
\Rightarrow R^{*}=R-\{\varnothing\}=\{\{a\},\{b\},\{c\},\{a, b\},\{a, c\},\{b, c\}, X\}
$$




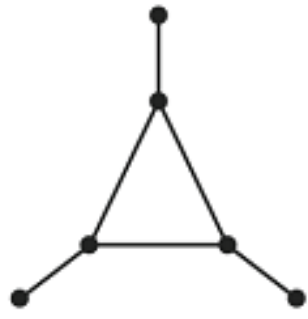

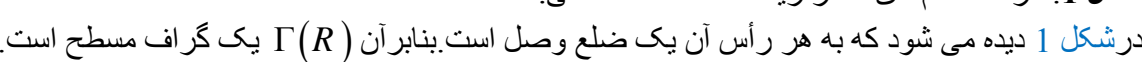

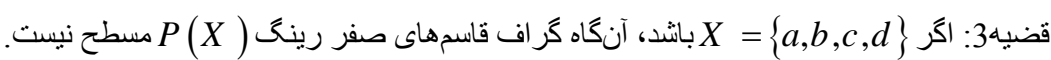

$$
\begin{array}{r}
R=P(X)=\left\{\begin{array}{l}
\{a\},\{b\},\{c\},\{d\},\{a, b\},\{a, c\}, \\
\{a, d\},\{b, c\},\{b, d\},\{c, d\},\{a, b, c\}, \\
\{a, b, d\}\{a, c, d\},\{b, c, d\}, X, \varnothing
\end{array}\right\} \\
\Rightarrow R^{*}=R-\{\varnothing\}=\left\{\begin{array}{l}
\{a\},\{b\},\{c\},\{d\},\{a, b\},\{a, c\}, \\
\{a, d\},\{b, c\},\{b, d\},\{c, d\},\{a, b, c\}, \\
\{a, b, d\},\{a, c, d\},\{b, c, d\}, X
\end{array}\right\}
\end{array}
$$

فرضكند

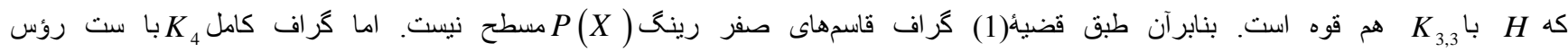

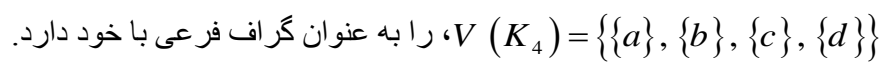

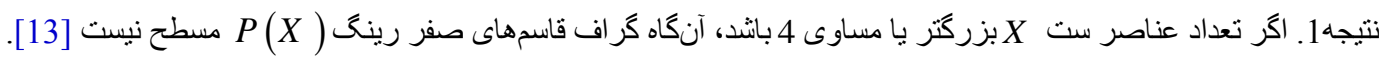

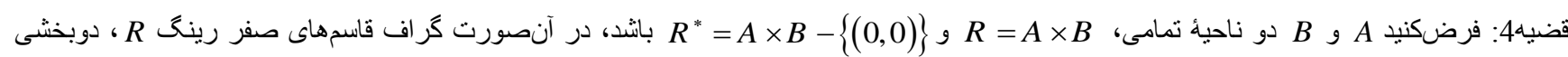

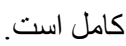
اثبات: فرضكنيد رؤس آن |

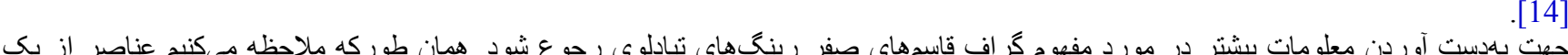

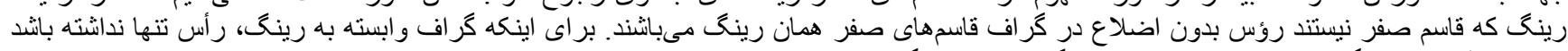

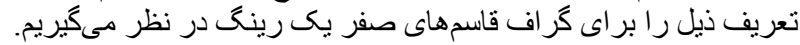

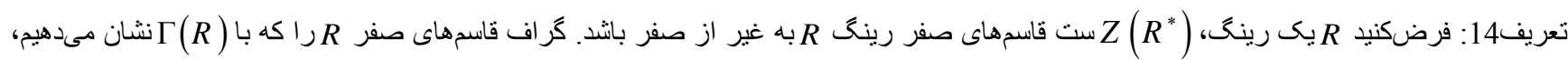

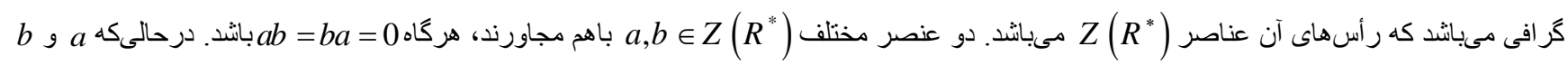

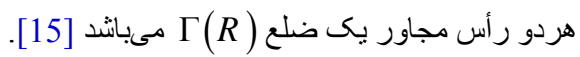

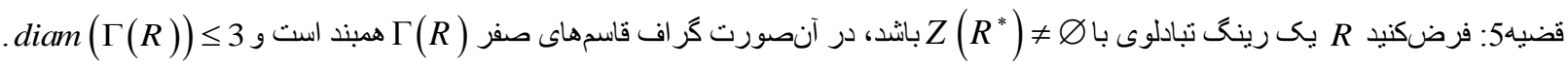
اثبات: فرضكنيد دو عنصر مختلف

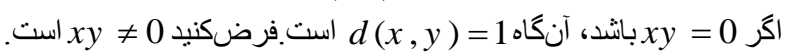
اكر اكر = 0 اكر $x^{2}=y^{2}$ باثد، آنكاه طبق تعريف (8)، اكر اكر 0 اكر

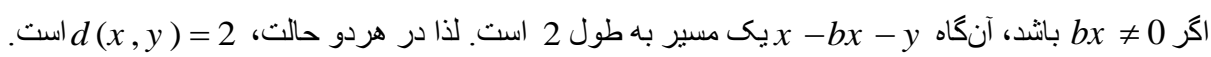

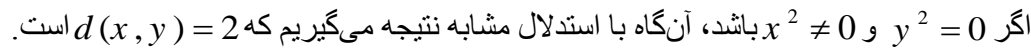
حال فرضكنيد كه $a x=b y=0$ ب $a, b \in Z\left(R^{*}\right)-\{x, y\}$ 


$$
\begin{aligned}
& \text { اكر b= a باتثد، آنكاه } \\
& \text { اكر } 0 \text { = } 0 \text { باثد، آنكاه } \\
& d(x, y) \leq 3
\end{aligned}
$$

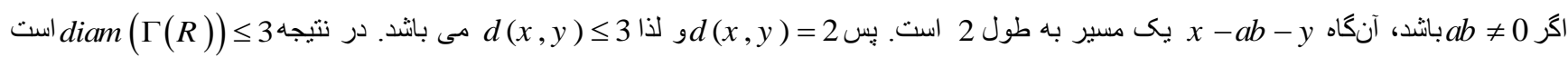

$$
\text { مثال1: اكر }
$$

$$
\begin{array}{r}
(\overline{0}, \overline{1})(\overline{0}, \overline{2}) \neq(\overline{0}, \overline{0}),(\overline{0}, \overline{1})(\overline{1}, \overline{2})=(\overline{0}, \overline{2}) \neq(\overline{0}, \overline{0}) \\
(\overline{1}, \overline{0})(\overline{1}, \overline{2}) \neq(\overline{0}, \overline{0})
\end{array}
$$$$
\text { از طرف (م) }
$$

$$
(\overline{0}, \overline{1})(\overline{1}, \overline{0})=(\overline{0}, \overline{0}),(\overline{1}, \overline{0})(\overline{0}, \overline{2})=(\overline{0}, \overline{0}),(\overline{0}, \overline{2})(\overline{1}, \overline{2})=(\overline{0}, \overline{0})
$$

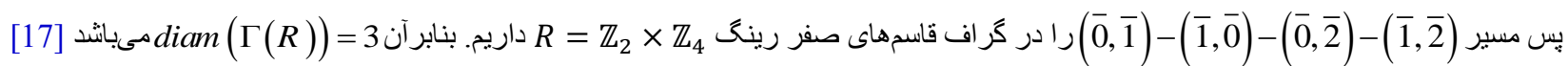

$$
\begin{aligned}
& \text { نتيجه2: فرضكنيد } \\
& \Gamma\left(Z_{2} \times F_{q}\right)=K_{1, q-1}, \Gamma\left(F_{p} \times F_{q}\right)=K_{p-1, q-1} \\
& \text { است [18] } \\
& \text { 3 }
\end{aligned}
$$

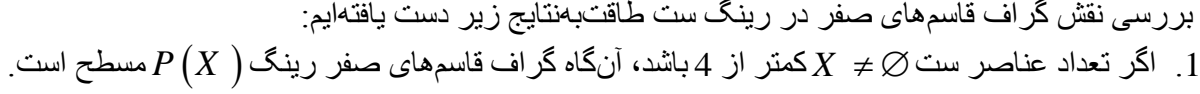

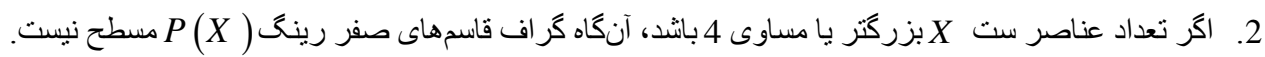

\section{References}

[1] J. Morty, Graph theory and its applications, translated by Dara Moazami, 4th ed. Tehran: University Publishing Center, 2006.

[2] Friendship, Graph theory and its applications, 3rd ed. Tehran: Mobtakaran, 1390.

[3] L. S. Schutz, Discrete mathematics, translated by Ali Akbar Alamzadeh, Shamshad Press. Tehran: Kourosh, 1374.

[4] Nazari, "The role of graph theory in the ring factor," Kabul University Scientific Journal, vol. 3, p. 182, 2015.

[5] Nazari, "Graph of zero qasems in the rings, scientific Journal of Takhar University, ," Research Work for Promotion to the Scientific Rank of the University, pp. 67-68, 2015.

[6] Theoretical, "The role of proximity, qccurrence, laplacein and asymptomatic laplacein in graph theory," Kabul University Scientific Journal, vol. 3, p. 123, 2016.

[7] Theoretical, "The importance of graph theory in mathematics," Scientific Journal of Takhar University, vol. 2, pp. 81- 88, 1396.

[8] A. Aleve's, "Graf divisor de zero de um Anal Commutative Claudia Juliana Finely gone," pp. 18-23, 2011.

[9] L. Bingjun, "Zero-divisor graph of triangular matrix rings over commutative rings1," International Journal of Algebra, vol. 5, pp. 255-260, 2011.

[10] B. Z. Inane and P. M. Zofran, "Zero-divisor graphs of matrices over com- mutative ring," Comm, Alg, pp. 11-32, 2009.

[11] D. F. Anderson, R. Levy, and J. Shapiro, "Zero-divisor graphs, von Neumann regular rings, and Boolean algebras," Journal of Pure and Applied Algebra, vol. 180, pp. 221-241, 2003.

[12] D. Cretkovic, M. Droob, and H. Sachs, "Spectra of graphs, theory and applications," 3rd ed. Heidelberg -Leipzig,: Johann Ambrosias Barth Verlag, 1995.

[13] K. Balinska, M. Kupczyk, S. Simic, and K. Zwierzynski, "On generating all integral graphs on 11 vertices," Computer Science Center Report, pp. 1-36, 2000.

[14] L. Kung-chug and P. Robert, "Ruthanne keego graph theory translation by Joanne Tammie," pp. 1-6, 2008.

[15] L. Vadis, "Lecture notes on graph theory," pp. 7-44, 2009.

[16] T. Lucas, "The diameter of a zero-divisor Graph, J. Algebra," vol. 10, 2006.

[17] V. K. Bhatt and R. Rania, "A not on zero-divisor graph over rings," J. Contempt. Math, Sci, vol. 174, 2007.

[18] W. U. Tongue, "On directed zero-divisor graphs of finite rings," Discrete Mathematics, p. 46, 2005. 